\title{
GROUPS WITH THE SAME LOWER CENTRAL SEQUENCE AS A RELATIVELY FREE GROUP. I THE GROUPS
}

\author{
BY \\ GILBERT BAUMSLAG $\left({ }^{1}\right)$
}

1. Introduction. 1.1. There is an old conjecture that a group of cohomological dimension one is free. This paper and the ones that will follow it are, in part, an outgrowth of a desire to make a contribution towards this conjecture. In this we have had little discernible success. However the approach we have taken has led to classes of groups which seem to be of considerable interest for their own sake. It is these groups that are the subject matter of this series of papers. The purpose of this one, having put these groups into their proper perspective, is to establish the existence of many groups of this kind. In the second and third papers we shall investigate some of their properties and their relationship with groups whose second integral homology groups $\left({ }^{2}\right)$ are zero.

1.2. Let $\mathfrak{B}$ be a variety of groups. P. Hall [12] has termed a group $S$ a splitting group for $\mathfrak{B}$ if

(i) $S$ is a $\mathfrak{B}$-group, i.e., $S \in \mathfrak{B}$, and

(ii) every exact sequence

$$
1 \rightarrow K \rightarrow E \rightarrow S \rightarrow 1
$$

of $\mathfrak{B}$-groups splits. It is clear that a free $\mathfrak{B}$-group is a splitting group for $\mathfrak{B}$. There are, conversely, very few varieties for which it is known that the splitting groups are precisely the free groups in the variety. Essentially, the only result in this direction is that in a nilpotent variety of exponent zero every splitting group is free (P. Hall [12]). In his paper [12] P. Hall asks whether, more generally, a splitting group in an arbitrary variety of exponent zero is free. This seems to be a very difficult question.

Now it is not difficult to prove

Proposition A. Let $G$ be a group, let $N$ be a normal subgroup of $G$ and let $C$ be a complement for $N$ in $G$, i.e.,

$$
N \cap C=1, \quad N C=G .
$$

Let further $\mathfrak{B}$ be a variety of groups. Then $C / V(C)$ is a complement of some normal subgroup of $G / V(G)$, where here $V(G)$ is the verbal subgroup of $G$ corresponding to

Received by the editors November 19, 1965.

(') Alfred P. Sloan Fellow.

$\left({ }^{2}\right)$ See [17]. 
the variety $\mathfrak{B}$ (i.e., $V(G)$ is the intersection of those normal subgroups of $G$ with factor group in $\mathfrak{B})$.

It follows that if $S$ is a splitting group for $\mathfrak{B}$, then $S / S_{k}$ is a splitting group for the variety $\mathfrak{B}(k)$ of all $\mathfrak{B}$-groups which are nilpotent of class at most $k$ for $k=1,2,3, \ldots$ (here $S_{k}$ is the $k$ th term of the lower central series of $S$, beginning with $S_{1}=S$ ). So if $\mathfrak{B}$ is a variety of exponent zero, it follows from $\mathrm{P}$. Hall's theorem that $S / S_{k}$ is free in the variety $\mathfrak{B}(k)$. Let us term

$$
S / S_{2}, S / S_{3}, \ldots
$$

the lower central sequence of the group $S$. If

$$
T / T_{2}, T / T_{3}, \ldots
$$

is the lower central sequence of $T$, then we say $S$ and $T$ have the same lower central sequence if there are isomorphisms $\theta_{k}$ from $S / S_{k}$ to $T / T_{k}$ such that $\theta_{k}$ induces $\theta_{k-1}$ on $S / S_{k-1}$ to $T / T_{k-1}$, for $k=2,3, \ldots$. Our remarks above show that if $\mathfrak{B}$ is a variety of exponent zero, then a splitting group for $\mathfrak{B}$ has the same lower central sequence as a suitably chosen free $\mathfrak{B}$-group (cf. pp. 345-346 and the proof of Theorem 2 of P. Hall [12], and K. W. Gruenberg [11]). Now an $m$-generator group with the same lower central sequence as a residually nilpotent, relatively free group of finite rank $m$ is free (W. Magnus [19]). So if $\mathfrak{B}$ is a variety of exponent zero in which the free groups are residually nilpotent, then an $m$-generator splitting group $S$ for $\mathfrak{B}$, whose factor derived group $S / S_{2}$ cannot be generated by fewer than $m$ elements, is free (P. Hall [12]). This line of reasoning suggests that a residually nilpotent group with the same lower central sequence as a residually nilpotent and relatively free group is free. The main purpose of this paper is to establish the existence of nonfree groups of this kind.

1.3. In order to explain our results let us term a variety $\mathfrak{M}$ a Magnus variety if

(a) every free $\mathfrak{M}$-group is residually nilpotent, and

(b) the lower central factors $F_{k} / F_{k+1}$ of every free $\mathfrak{M}$-group are torsion-free, for $k=1,2,3, \ldots$.

The Magnus varieties include, in particular, the variety of all groups (W. Magnus [18] and E. Witt [24]) and the polynilpotent varieties of all polynilpotent groups of a given class row (K. W. Gruenberg [10]). A group $P$ in a variety $\mathfrak{M}$ will be termed para $\mathfrak{M}$-free or parafree in $\mathfrak{M}$ or, if $\mathfrak{M}$ is understood to be the variety involved, simply parafree, if

(i) $P$ is residually nilpotent, and

(ii) $P$ has the same lower central sequence as some free group in $\mathfrak{M}$.

If we denote by $\mu(g)$ the minimal number of generators of a group $G$, then we define $\rho(G)$, the rank of $G$, by

$$
\rho(G)=\mu\left(G / G_{2}\right),
$$

and the deviation $\delta(G)$ of $G$ by

$$
\delta(G) \rho(G=\mu(G)-) .
$$


Then we shall prove in $\S 3$ the

THEOREM $1\left(^{3}\right)$. In the variety of all groups there exist for every pair of integers $r$ and $d$ satisfying $r \geqq 2$ and $d \geqq 1$ infinitely many parafree groups of rank $r$ and deviation $d$.

Theorem 1 contrasts with the following rather weaker result, which will be proved in $\$ 4$.

THEOREM 2. Let $\mathfrak{M}$ be a Magnus variety which contains $\mathfrak{A}^{2}$, the variety of all metabelian groups. Then there exists for every integer $r \geqq 2$ a parafree group of rank $r$ which is not free.

Theorem 2 can be sharpened to

THEOREM $2^{\#}$. Let $\mathfrak{M}$ be a Magnus variety which contains $\mathfrak{A}^{2}$. Then there exists for every pair of integers $r \geqq 2$ and $d \geqq 1$ a parafree group of rank $r$ and deviation $d$.

However the added labor involved does not seem to merit including this version here. Even this version of Theorem 2 compares unfavorably with Theorem 1 . This is not surprising in view of our meagre knowledge of varieties. A glance at the proofs will substantiate this remark.

1.4. Theorem 1 and Theorem 2 leave open a large number of questions. For example, can a parafree group be of finite rank and yet have infinite deviation? These and other questions we shall consider elsewhere.

There is, however, a possible offshoot of Theorem 2 which should be mentioned. Theorem 2 provides us with parafree groups which are not free. But, for example, is every parafree group in $\mathfrak{A}^{2}$ a splitting group? If this is the case, Theorem 2 contains a negative answer to Philip Hall's question about splitting groups.

Theorem 1 and Theorem 2 also provide a spectacular illustration of the hopelessness of trying to solve the isomorphism problem of even the best behaved groups via the lower central sequence (cf. [8]).

1.5. The relevance of splitting groups to groups of cohomological dimension one $\left({ }^{4}\right)$ is not hard to understand. It is clear that a group $G$ is of cohomological dimension one if and only if every exact sequence

$$
1 \rightarrow A \rightarrow E \rightarrow G \rightarrow 1
$$

with $A$ soluble, splits. This implies, on making use of Proposition A, that for every soluble variety $\mathfrak{B}, G / V(G)$ is a splitting group for $\mathfrak{B}$. It follows from this observation that every residually soluble group of cohomological dimension one is parafree (G. Baumslag and K. W. Gruenberg [7]).

( $\left.{ }^{3}\right)$ The first example of this kind was described by the author in [5].

$\left({ }^{4}\right)$ See [17], pages 103-124. 
Further knowledge of splitting groups in soluble varieties may, in this way, provide us with more information about residually soluble groups of cohomological dimension one, perhaps leading even to a proof that residually soluble groups of cohomological dimension one are free.

\section{A useful proposition.}

2.1. The object of this section is to prove the following proposition and its corollary.

Proposition 1. Let $G$ be a finitely generated group in a variety $\mathfrak{B}$. Suppose $r$ is the rank of $G$. If $G / G_{n+1}$ contains as a subgroup a free group in $\mathfrak{B}(n)$ of rank $r$ and class $n$ for every $n$, then $G$ has the same lower central sequence as a free group in $\mathfrak{B}$.

Proof. An $r$-generator group in $\mathfrak{B}(n)$ with a free $\mathfrak{B}(n)$-subgroup of rank $r$ is free ([3]). So Proposition 1 follows.

This leads us to the important

Corollary 1. Let $\mathfrak{M}$ be a Magnus variety, $P$ a group in $\mathfrak{M}$. Let

$$
Q=\operatorname{gp}(P, u)
$$

be a supergroup of $P$ contained in $\mathfrak{M}$ such that

(i) $Q$ is residually nilpotent,

(ii) there are elements $a_{1}, \ldots, a_{r}$ of $P$ which freely generate $P$ modulo $P_{2}$ such that

$$
u^{\lambda}=a_{1}^{\lambda_{1}} \cdots a_{r}^{\lambda} \bmod Q_{2} \quad(\lambda \neq 0)
$$

where at least one of the $\lambda_{j}$ is nonzero and no prime divisor of $\lambda$ is a divisor of every $\lambda_{j}$,

(iii) $Q / Q_{n+1}$ contains a free $\mathfrak{M}_{n}$-group of rank $r$ and class $n$ for every $n$.

Then $Q$ is parafree of rank $r$.

Corollary 1 follows immediately from Proposition 1 since, by (ii), $Q / Q_{2}$ is an $r$-generator group.

The point of Corollary 1 is that if we adjoin roots to parafree groups in the right way we will get further parafree groups.

\section{The proof of Theorem 1.}

3.1. Corollary 1 suggests a way of producing parafree groups. We push this a little further by proving

PROPOSITION 2. Let $r$ and $n$ be positive integers, let $H$ be parafree of rank $r$ and let $(x)$ be the infinite cyclic group on $x$. Let, further, $h \in H$ and let $G$ be the generalized free product of $H$ and $(x)$ identifying $h$ with $x^{n}$ :

$$
G=\left\{H *(x) ; h=x^{n}\right\} .
$$

Suppose $h$ is an lth power of $h^{\prime}$ modulo $H_{2}$, where $h^{\prime}$ is itself not a power modulo $\mathrm{H}_{2}$. If $l$ and $n$ are coprime and if $G$ is residually nilpotent, then $G$ is parafree of rank $r$. 
The proof of Proposition 2 is easy. To see this observe that $H / H_{c+1}$ is free nilpotent of rank $r$. So, by a theorem of A. I. Mal'cev [20] it can be enlarged to a torsion-free nilpotent group in which $h H_{c+1}$ has an $n$th root $x_{c+1}$, say. Clearly $\mathrm{gp}\left(H / H_{c+1}, x_{c+1}\right)$ is a homomorphic image of $G$. It follows that $G / G_{c+1}$ contains a subgroup isomorphic to $H / H_{c+1}$ for every $c=1,2, \ldots$ So Corollary 1 applies and $G$ is parafree of rank $r$.

3.2. The crucial condition in Proposition 2 is that a certain generalized free product is residually nilpotent. So, in order to be able to apply Proposition 2 we must provide ourselves with a number of generalized free products which are residually nilpotent. This is the point of the following:

LEMMA 1. Let the finitely generated group $H$ be residually torsion-free nilpotent and let $H_{i}$ be an indexed copy of $H$ for each $i=1,2, \ldots, n(n<\infty)$ (thus the mapping $h \rightarrow h_{i}$ is an isomorphism between $H$ and $H_{i}$ for all relevant $i(h \in H)$ ). Suppose $u \in H$ generates its centralizer in $H$. Then the generalized free product

$$
P=\left\{H_{1} * H_{2} * \cdots * H_{n} ; u_{1}=u_{2}=\cdots=u_{n}\right\}
$$

is residually a finite p-group for every prime $p$.

Proof. Let $p$ be any prime and let $a \in P(a \neq 1)$. If $a$ is of length 0 or 1 then the homomorphisms which take $H_{i}$ to $H$ as follows

$$
h_{i} \rightarrow h \quad(i=1,2, \ldots, n),
$$

can be continued to a homomorphism $\theta$ of $P$ onto $H$. Notice that $a \theta \neq 1$. Since $H$ is finitely generated and residually torsion-free nilpotent, $H$ is residually a finite $p$-group (K. W. Gruenberg [10]). So there is a normal subgroup of $P$ of $p$-power index not containing $a$.

Suppose then that

$$
a=h_{i_{1}}^{(1)} h_{i_{2}}^{(2)} \cdots h_{i_{l}}^{(l)}
$$

is of length $l$, the factors $h_{i j}^{(j)}$ coming strictly out of alternate constituents $H_{i,}$. This means that $h^{(j)} \notin \mathrm{gp}(u)$ for $j=1, \ldots, l$. Hence $\left[h^{(j)}, u\right] \neq 1$, since $u$ generates its centralizer $\left({ }^{5}\right)$. It follows that we can find a normal subgroup $N$ of $H$ of $p$-power index so that $\left[h^{(j)}, u\right] \notin N$ for $j=1, \ldots, l$. Consequently

$$
h^{(j)} \dot{N} \notin \operatorname{gp}(u N) \quad(j=1, \ldots, l) .
$$

Now denote the image of $N$ in $H_{i}$ by $N_{i}$. Form

$$
Q=\left\{H_{1} / N_{1} * H_{2} / N_{2} * \cdots * H_{l} / N_{l} ; u_{1} N_{1}=u_{2} N_{2}=\cdots=u_{l} N_{l}\right\} .
$$

It follows from (1) that if $\sigma$ is the homomorphism of $P$ onto $Q$ which is defined as the extension of the natural homomorphisms from $H_{i}$ to $H_{i} / N_{i}$ for each $i$, then

$$
a \sigma=h_{i_{1}}^{(1)} N_{i_{1}} \cdots h_{i_{l}}^{(l)} N_{i_{l}} \neq 1 \text {. }
$$

$\left.{ }^{5}\right)[x, y]$ denotes the commutator $x^{-1} y^{-1} x y$. 
Observe that $Q$ is a generalized free product of finite isomorphic $p$-groups with a cyclic subgroup amalgamated. Now in this case the isomorphisms from $H_{i} / N_{i}$ to $H / N$ defined by $h_{i} N_{i} \rightarrow h N$ can be continued to a homomorphism $\phi$ of $Q$ onto $H / N$. Let $K$ be the kernel of $\phi$. Then clearly $Q / K$ is a finite $p$-group and

$$
K \cap H_{i} / N_{i}=1 \quad(i=1,2, \ldots, l) .
$$

So by a theorem of $\mathrm{H}$. Neumann [22], $K$ is free. Consequently $Q$ is residually a finite $p$-group, since an extension of a residually finite $p$-group by a finite $p$-group is residually a finite $p$-group, the main point here being that free groups are residually finite p-groups (K. Iwasawa [14]). So once more there is a normal subgroup of $p$-power index not containing $a$ in $P$, since there is such a normal subgroup in $Q$ not containing $a \sigma$. This completes the proof of Lemma 1 .

Corollary 2. Let $H$ and $u \in H$ satisfy the hypotheses of Lemma 1. Then for every prime $p$ and every infinite cyclic group $(x)$ generated by $x$, the generalized free product

$$
\left\{H *(x) ; u=x^{p}\right\}
$$

is residually a finite p-group.

Proof. Let $H_{i}=x^{-i} H x^{i}(i=0,1, \ldots, p-1)$. If $P$ is the subgroup of

$$
\left\{H *(x) ; u=x^{p}\right\}
$$

generated by $H_{0}, H_{1}, \ldots, H_{p-1}$, then

$$
P=\left\{H_{0} * H_{1} * \cdots * H_{p-1} ; u_{0}=u_{1}=\cdots=u_{p-1}\right\},
$$

where $u_{i}=x^{-i} u x^{i}$. So by Lemma $1 P$ is residually a finite $p$-group. But $P$ is a normal subgroup of $\left\{H *(x) ; u=x^{p}\right\}$ of index $p$; therefore $\left\{H *(x) ; u=x^{p}\right\}$ is residually a finite $p$-group.

3.3. We are now in a position to apply Proposition 2 so as to obtain

PROPOSITION 3. Let $H$ be parafree of rank $r$, let $(x)$ be the infinite cyclic group on $x$, let $h \in H$ and let $l$ be a positive integer which is not divisible by the prime $n$. If $h$ generates its centralizer in $H$ and if $h=h_{1}^{l}$ modulo $H_{2}$, where $h_{1}$ is not a proper power modulo $\mathrm{H}_{2}$, then

$$
G=\left\{H *(x) ; h=x^{n}\right\}
$$

is parafree of rank $r$.

Proof. By Corollary 2, $G$ is residually nilpotent. So Proposition 2 applies, ensuring that $G$ is parafree of rank $r$, as desired.

3.4. Before proceeding to the proof of Theorem 1, which will actually be accomplished by making use of Proposition 3, we require some facts about some of the following groups.

LEMMA 2. Let

$$
G=\left(a, b, \ldots, c, x, y, \ldots, z ; a^{l} b^{m}=x^{n}, a^{p} b^{q}=y^{r}, \ldots, a^{s} b^{t}=z^{u}\right),
$$


where the integers $l, m, n, p, q, r, \ldots, s, t, u$ are all greater than 1 and $l, p, \ldots, s$ are distinct. If $v$ is a positive integer such that $v \notin\{l, p, \ldots, s\}$, then for every nonzero integer $w$ the centralizer $C\left(a^{v} b^{w} ; G\right)$ of $a^{v} b^{w}$ in $G$ is generated by $a^{v} b^{w}$ :

$$
C\left(a^{v} b^{w} ; G\right)=\operatorname{gp}\left(a^{v} b^{w}\right) .
$$

Proof. The proof is by induction on the number $k$ of elements in $\{x, y, \ldots, z\}$. If $k=1$ then $G$ is simply the generalized free product of the free group $F$ on $a, b, \ldots, c$ and the infinite cyclic group $(x)$ on $x$ amalgamating $a^{l} b^{m}$ and $x^{n}$ :

$$
G=\left\{F *(x) ; a^{l} b^{m}=x^{n}\right\} .
$$

Notice that $a^{l} b^{m}$ generates its centralizer in $F$ and that $a^{v} b^{w}$ is not conjugate to an element in gp $\left(a^{l} b^{m}\right)$. So by Lemma 28.2 of [2],

$$
C\left(a^{v} b^{w} ; F\right)=C\left(a^{v} b^{w} ; G\right)
$$

but $C\left(a^{v} b^{w} ; F\right)=\mathrm{gp}\left(a^{v} b^{w}\right)$ and the result follows.

Suppose now that $k>1$. Put

$$
H=\mathrm{gp}(a, b, \ldots, c, x, y, \ldots) ;
$$

thus $H$ is generated by all of the generators of $G$ excluding $z$. Clearly,

$$
G=\left\{H *(z) ; a^{s} b^{t}=z^{u}\right\}
$$

Now it follows from the fact that the integers $l, p, \ldots, s, v$ are all distinct that each of the elements $a^{l} b^{m}, a^{p} b^{q}, \ldots, a^{s} b^{t}, a^{v} b^{w}$ generate their centralizers in $F$. By induction then we find

$$
C\left(a^{s} b^{t} ; H\right)=\mathrm{gp}\left(a^{s} b^{t}\right) \text { and } C\left(a^{v} b^{w} ; H\right)=\mathrm{gp}\left(a^{v} b^{w}\right) .
$$

It follows also, as part of the induction hypothesis, that $H$ belongs to the class of groups $P_{\pi}$, where $\pi$ is the set of all primes (see p. 256, Theorem 29.1 and Lemma 28.2 of [2]). Moreover as $a^{v} b^{w}$ is not conjugate in $F$ to an element in $\mathrm{gp}\left(a^{s} b^{t}\right)$ it follows by a fairly easy induction that $a^{v} b^{w}$ is not conjugate in $H$ to an element in gp $\left(a^{s} b^{t}\right)$. Thus we may apply Theorem 29.1 of [2] to deduce that $G \in P_{\pi}$. It follows then from Lemma 28.2 of [2] that

$$
C\left(a^{v} b^{w} ; G\right)=C\left(a^{v} b^{w} ; H\right)=\operatorname{gp}\left(a^{v} b^{w}\right)
$$

is desired.

3.5. The second fact that we shall need can be formulated in rather more general terms than we choose to.

LemMA 3. Let $n, r, \ldots, u$ be distinct odd primes and let

$$
G=\left(a, b, \ldots, c, x, y, \ldots, z ; a^{2} b^{2}=x^{n}, a^{4} b^{4}=y^{r}, \ldots, a^{2^{k}} b^{2^{k}}=z^{u}\right),
$$

(where $k$ is the number of elements in $\{x, y, \ldots, z\}$ ). Then modulo $G_{2}, a^{2^{k+1}} b^{2^{k+1}}$ is the $2^{k+1} \cdot n \cdot r \cdots \cdot$ uth power of an element which is itself not a proper power. 
Proof. The crux of the matter is that if $A$ is an abelian group generated by two elements $f$ and $g$ such that $f^{v}=g^{w}$, where $v$ and $w$ are coprime positive integers, then $A$ is cyclic in a rather special way. In fact suppose we choose $\nu$ and $\mu$ so that $\nu v+\mu w=1$. Then

$$
f=\left(f^{u} g^{v}\right)^{w} \text { and } g=\left(f^{u} g^{v}\right)^{v}
$$

so $A$ is a cyclic group generated by $f^{\mu} g^{\nu}$. It follows from (2) that if $A$ is torsion-free then $f$ is an $i$ th power if and only if $i$ divides $w$. The complete proof of Lemma 3 follows without much difficulty from this observation.

3.6. The third fact we need is

LEMMA 4. Let

$$
G=\left(a, b, \ldots, c, x, y, \ldots, z ; a^{2} b^{2}=x^{n}, a^{4} b^{4}=y^{r}, \ldots, a^{2^{k}} b^{2^{k}}=z^{u}\right),
$$

where the integers $n, r, \ldots, u$ are all at least two. Then the minimal number of generators of $G$ is $j+k$ where $j$ is the number of elements in $\{a, b, \ldots, c\}$.

Proof. We add the further relations $a^{2}=b^{2}=1$ to $G$. The resultant factor group $\tilde{G}$ of $G$ is simply the free product of two groups of order two, a free group of rank $j-2$ and cyclic groups of orders $n, r, \ldots$ and $u$ respectively. By the GrushkoNeumann theorem (see e.g., A. G. Kurosh [16, Volume 2, p. 57]) $\tilde{G}$ is a $(j+k)$ generator group. So it follows immediately that $G$ is a $(j+k)$-generator group.

3.7. If $X$ is a group and $l$ is an integer, then we denote by $X^{l}$ the subgroup of $X$ generated by its $l$ th powers. This type of subgroup will be useful in the proof of the last fact we shall need in order to be able to prove Theorem 1.

LEMMA 5. Let

$$
G=\left(a, b, \ldots, c, x, y, \ldots, z ; a^{2} b^{2}=x^{m}, a^{4} b^{4}=y^{p}, \ldots, a^{2^{k}} b^{2^{k}}=z^{q}\right),
$$

and

$$
G^{*}=\left(a, b, \ldots, c, x, y, \ldots, z ; a^{2} b^{2}=x^{r}, a^{4} b^{4}=y^{s}, \ldots, a^{2^{k}} b^{2^{k}}=z^{t}\right),
$$

where $m, p, \ldots, q, r, s, \ldots, t$ are distinct odd primes. Then $G$ is not isomorphic to $G^{*}$.

Proof. We consider

$$
G / G^{2 q} \text { and } G^{*} / G^{* 2 q} \text {. }
$$

Notice that the primes $2, m, p, \ldots, q, r, s, \ldots, t$ are all distinct. So it follows that $G^{*} / G^{* 2 a}$ can be generated by $j$ elements (here $j$ is the number of elements in $\{a, b, \ldots, c\})$. However we claim that $G / G^{2 q}$ can not be generated by $j$ elements.

To see this consider the wreath product $\left({ }^{6}\right)$

$$
W=\left(C_{2} \times C_{2} \times \cdots \times \text { wr } C_{2}\right) C_{q}
$$

${ }^{(6)}$ See, e.g., [13]. 
of the direct product of $j$ groups of order two and a (cyclic) group of order $q . W$ is clearly a homomorphic image of $G / G^{2 q}$. In order to complete the proof of Lemma 5 , it is therefore enough to show that $W$ requires at least $j+1$ generators. Here a simple counting argument suffices since a $j$-generator subgroup of $W$ has order at most

$$
2 q 2^{q(j-1)}
$$

whereas $W$ itself has order

$$
q 2^{q j}
$$

3.8. We are now in a position to prove Theorem 1. To this end let $r$ be an integer at least 2, and let $m$ be an integer exceeding $r$. Put, for $i=1,2, \ldots$,

$$
\begin{aligned}
& G(i)=\left(a, b, \ldots, c, x, y, \ldots, z ; a^{2} b^{2}=x^{p_{t d}},\right. \\
& \left.a^{4} b^{4}=y^{p_{t d+1}}, \ldots, a^{2^{m-r}} b^{2^{m-r}}=z^{p_{t d+m-r}}\right),
\end{aligned}
$$

where $p_{1}, p_{2}, \ldots$ are the odd primes arranged in order of magnitude and the number of elements in $\{a, b, \ldots, c\}$ is $r$. By Lemma 4 each of the groups $G(i)$ requires precisely $m$ generators. Moreover by Lemma 5 no two of the $G(i)$ are isomorphic. Finally, it follows by induction, Lemma 2 and Lemma 3 that Proposition 3 applies. Hence each of the $G(i)$ is parafree of rank $r$ and the proof of Theorem 1 is complete.

3.9. Suppose now that we choose $r=2, m=3$ in 3.8. Then it follows easily either directly or by applying a theorem of D. B. A. Epstein [9] that the groups $G(i)$ are freely indecomposable.

We define now, for every properly increasing sequence

$$
\alpha: \alpha_{1}, \alpha_{2}, \ldots
$$

of positive integers, $G(\alpha)$ to be the free product of the groups $G\left(\alpha_{i}\right)$ :

$$
G(\alpha)=G\left(\alpha_{1}\right) * G\left(\alpha_{2}\right) * \cdots .
$$

It follows from A. I. Mal'cev [21] that $G(\alpha)$ is residually torsion-free nilpotent. By a variation of the arguments used to prove Proposition 3 it then follows that $G(\alpha)$ is parafree of rank $\aleph_{0}$. If $\beta: \beta_{1}, \beta_{2}, \ldots$ is another increasing sequence of positive integers, then $G(\alpha) \cong G(\beta)$ if and only if $\alpha=\beta$. For if $\alpha \neq \beta$ there is a first integer $i$ for which $\alpha_{i} \neq \beta_{i}$. If $\alpha_{i}<\beta_{i}$ then $G(\alpha)$ has precisely one free factor isomorphic to $G\left(\alpha_{i}\right)$; since the groups $G(j)$ are nonisomorphic, $G(\beta)$ does not have such a free factor (cf. R. Baer and F. W. Levi [1]); so $G(\alpha)$ is not isomorphic to $G(\beta)$. Similarly if $\alpha_{i}>\beta_{i}$ we have again that $G(\alpha)$ is not isomorphic to $G(\beta)$. Since the number of such sequences $\alpha$ is the power of the continuum, this means we have proved the following corollary of Theorem 1.

COROLlaRY 3. There are at least continuously many parafree groups of rank $\boldsymbol{\aleph}_{0}$. Corollary 3 is clearly only part of a longer story. 


\section{The proof of Theorem 2.}

4.1. We start out by trying to apply Corollary 1 . Our aim is to prove that there exist nonfree parafree groups in every Magnus variety $\mathfrak{M}$ containing $\mathfrak{A}^{2}$, of every rank. With this in mind, let $F$ be a finitely generated free group in $\mathfrak{M}$ freely generated by the $r$ elements

$$
a, b, b^{\prime}, \ldots, c \text {. }
$$

Our procedure in the variety of all groups is to make use of generalized free products. But this is impossible in an arbitrary variety $\mathfrak{M}$ since very little is known about the existence of such generalized products (see e.g., J. Wiegold [23]). We shall construct a nonfree parafree group $G$ with the same lower central sequence as $F$ by adjoining a cube root of $a^{2} b^{2}$ to $F$.

Now $F$ is residually nilpotent. So $F$ is a subdirect product of the groups $F / F_{i}$; i.e., there is a monomorphism $\phi$ of $F$ into the cartesian product $P$ of the factor groups $F / F_{i}$ of $F$, such that the projection $\pi_{i}$ of $P$ onto $F / F_{i}$ maps $F \phi$ onto $F / F_{i}$ :

$$
\phi: F \rightarrow P=\prod_{i=1}^{\infty} F / F_{i} .
$$

Henceforth, we identify $F$ with its image $F \phi$ in $P$.

Consider now the element $a^{2} b^{2}$ in $F$. In order to adjoin a cube root of $a^{2} b^{2}$ to $F$ we adjoin a cube root of $\left(a^{2} b^{2}\right) \pi_{i}$ to $F / F_{i}$ for each $i$. A little care is necessary here. Thus we recall that a torsion-free nilpotent group can be embedded in a minimal torsion-free nilpotent divisible group lying in the same variety (see [20] and [6, Proposition 2]). Now $F / F_{i}$ is torsion-free nilpotent and clearly lies in $\mathfrak{M}$. So we can choose a minimal, torsion-free nilpotent, divisible supergroup $G(i)$ containing $F / F_{i}$, which lies in $\mathfrak{M} . G(i)$ contains a (unique) cube root $d_{i}$ of $\left(a^{2} b^{2}\right) \pi_{i}$. Let

$$
Q=\prod_{i=1} G(i) \text {. }
$$

Obviously then (cf. (4) and (5)) $a^{2} b^{2}$ has a cube root $d$, say, in $Q$. We put

$$
G=\mathrm{gp}(F, d) \text {. }
$$

4.2. Clearly $G$ is residually nilpotent and lies in $\mathfrak{M}$. Since

$$
a^{2} b^{2}=d^{3},
$$

it is clear that $G / G_{2}$ can be generated by $r$ elements. This means that $G$ satisfies the conditions laid down by Corollary 1 . So $G$ is parafree in $\mathfrak{M}$ of rank $r$.

4.3. It remains to prove, and this turns out to be a little awkward, that $F$ and $G$ are not isomorphic. It is obvious that $G$ can be generated by $r+1$ elements; in fact, we shall prove that $G$ can not be generated by $r$ elements, ensuring that $F$ and $G$ are not isomorphic. The proof will be facilitated by making use of the following proposition (which can be formulated in rather more general terms, cf. Proposition 1 and the proof of the corollary on page 274 of [4]); the argument is essentially that of [4] and is therefore omitted. 
Proposition 4. Let $H \in \mathfrak{M}$ be residually a finite 2-group and let $\zeta$ be a mapping of (cf. (3))

$$
\{a, b, \ldots, c\}
$$

into $H$. If $(a \zeta)^{2}(b \zeta)^{2}$ has a cube root $e$, say, in $H$, then $\zeta$ can be extended to a homomorphism of $G$ into $H$ taking $d$ to $e$.

4.4. Our aim now is to use the above proposition to produce an $(r+1)$-generator homomorphic image of $G$.

To this end let $D$ be the free abelian group with basis

(6) $z ; \ldots, a_{1,-1}, a_{1,0}, a_{1,1}, \ldots ; \ldots, a_{2,-1}, a_{2,0}, a_{2,1}, \ldots ; \ldots, a_{m,-1}, a_{m, 0}, a_{m, 1}, \ldots$

The mapping $\psi$ of this basis of $D$ given by

$$
a_{i, j} \psi=a_{i, j+1} \quad(1 \leqq i \leqq m, j=0, \pm 1, \pm 2, \ldots), \quad z \psi=a_{1,0}^{3} z^{-1}
$$

clearly defines an automorphism, which we again denote by $\psi$, of $D$, of infinite order. Let $E$ be the splitting extension of $D$ by this automorphism $\psi$ :

$$
E=\operatorname{gp}\left(D, x ; x^{-1} d x=d \psi(d \in D)\right) .
$$

Thus $D$ is a normal subgroup of $E$ and

$$
E / D \text { is free cyclic on } x D \text {. }
$$

Moreover

$$
E=\operatorname{gp}\left(x, a_{1,0}, a_{2,0}, \ldots, a_{m, 0}, z\right) ;
$$

so $E$ can be generated by $m+2$ elements. It is important to notice that

$$
x^{-2}(x z)^{2}=x^{-2} x^{2} x^{-1} z x z=a_{1,0}^{3} z^{-1} z=a_{1,0}^{3} .
$$

We shall show that if $m=r-1$, then $E$ is the desired $(r+1)$-generator homomorphic image of $G$.

4.5. We need two facts about $E$. The first of these is that $E$ is residually a finite 2-group, while the second is that $E$ is not an $(m+1)$-generator group.

First we prove

LeMMA 6. $E$ is residually a finite 2-group.

Proof. By (8) $E / D$ is free cyclic. So in proving that $E$ is residually a finite 2-group it is enough to show that any given element $u \in D(u \neq 1)$ can be omitted from a normal subgroup of $E$ of 2-power index.

Notice now that $u$ lies in a finitely generated subgroup of $D$. In fact, without loss of generality, we may assume that

$$
u \in \operatorname{gp}\left(a_{1,0}, \ldots, a_{1, s}, a_{2,0}, \ldots, a_{2, s}, \ldots, a_{m, 0}, \ldots, a_{m, s}, z\right)
$$

where $s$ is of the form

$$
s=2^{t}-1
$$


We observe next that for some $k=2^{l}(l \geqq 1)$

$$
u \notin \operatorname{gp}\left(d^{k} \mid d \in D\right)=D^{k} .
$$

The idea now is to make certain that if we factor out $D^{k}$ and some large 2-power of $x$, then the result is a factor group of $E$ of 2-power order in which the canonical image of $u$ is not 1 . With this in mind, let $\tilde{D}$ be a direct product of $m 2^{t}+1$ cyclic groups of order $2^{l}$ with basis $\tilde{a}_{i j}, \tilde{z}$ :

$$
\tilde{D}=\operatorname{gp}\left(\tilde{a}_{i, j}, \tilde{z}\left(i=1,2, \ldots, m, j=0,1, \ldots, 2^{t}-1\right)\right) .
$$

Observe that the mapping

$$
\begin{array}{ll}
\tilde{\psi}: \tilde{a}_{i, j} \rightarrow \tilde{a}_{i, j+1} & \text { if } j+1 \leqq 2^{t}-1, \\
\tilde{\psi}: \tilde{a}_{i, j} \rightarrow \tilde{a}_{i, 0} & \text { if } j+1=2^{t}, \\
\tilde{\psi}: \tilde{z} \rightarrow \tilde{a}_{1,0}^{3} \tilde{z}^{-1} &
\end{array}
$$

defines an automorphism again denoted by $\tilde{\psi}$ of $\tilde{D}$.

Notice now that $\tilde{\psi}$ is of order a power of 2 . To see this, first observe that $\tilde{\alpha}^{s+1}$ leaves every $\tilde{\psi}_{i, j}$ fixed. But a simple calculation shows that (cf. (15))

$$
\tilde{z} \tilde{\psi}^{s+1}=\tilde{a}_{1,0}^{-3} \tilde{a}_{1,1}^{3} \ldots \tilde{a}_{1, s-1}^{-3} \tilde{a}_{1, s}^{3} \tilde{z}=p \tilde{z},
$$

where

$$
p=\tilde{a}_{1,0}^{-3} \tilde{a}_{1,1}^{3} \ldots \tilde{a}_{1, s-1}^{-3} \tilde{a}_{1, s}^{3} .
$$

It is clear, therefore, that after repeated use of (16)

$$
\tilde{z} \tilde{\psi}^{(s+1) k}=p^{k} \tilde{z}=\tilde{z} .
$$

So $\tilde{\psi}$ has order $(s+1) k=2^{t} 2^{l}=2^{t+l}$, as desired.

Let then $\tilde{E}$ be the splitting extension of $\tilde{D}$ by $\tilde{\psi}$ :

$$
\tilde{E}=\operatorname{gp}\left(\tilde{D}, \tilde{x} ; \tilde{x}^{-1} \tilde{d} \tilde{x}=\tilde{d} \tilde{\psi}(\tilde{d} \in \tilde{D}), \tilde{x}^{(s+1) k}=1\right) .
$$

Consider the mapping

$$
\sigma: x \rightarrow \tilde{x}, a_{1,0} \rightarrow \tilde{a}_{1,0}, \ldots, a_{m, 0} \rightarrow \tilde{a}_{m, 0}, z \rightarrow \tilde{z} .
$$

Since the relations satisfied by $x, a_{1,0}, \ldots, a_{m, 0}, z$ are also satisfied by

$$
\tilde{x}, \tilde{a}_{1,0}, \ldots, \tilde{a}_{m, 0}, \tilde{z}
$$

(cf. (6), (7), (15)) $\sigma$ defines a homomorphism $\tau$ of $E$ onto $\tilde{E}$. It follows on noting (11), (12), (13) that

$$
u \tau \neq 1 \text {. }
$$

But $\tilde{E}$ is a finite 2-group. This means that $E$ is residually a finite 2-group as claimed.

4.6. Second we prove

LEMMA 7. The minimum number of generators of $E$ is $m+2$. 
Proof. It suffices to show that $E$ has a subgroup $F$ of index 2 which cannot be generated by fewer than $2 m+2$ elements. For if $E$ could be generated by $m+1$ elements every subgroup of index 2 could be generated by

$$
2 m+1<2 m+2
$$

elements, since a subgroup of index $j$ of a free group of rank $n$ is of rank $1+j(n-1)$ (see A. G. Kurosh [16, Vol. 2, p. 37]).

Let then $E^{*}$ be the normal closure in $E$ of

Clearly (cf. (7))

$$
x^{2}, a_{1,0}, a_{2,0}, \ldots, a_{m, 0}, z
$$

$$
E^{*}=\operatorname{gp}\left(x^{2}, a_{1,0}, a_{1,1}, a_{2,0}, a_{2,1}, \ldots, a_{m, 0}, a_{m, 1}, z\right) .
$$

In order to find a complete set of defining relations for $E^{*}$ it is necessary to give a complete set of defining relations for $E$. For example, the relations

$$
\left[x^{-s} a_{i, 0} x^{s}, a_{j, 0}\right]=1, \quad\left[x^{-s} a_{i, 0} x^{s}, z\right]=1, \quad x^{-1} z x=a_{1,0}^{3} z^{-1}
$$

where $s=0, \pm 1, \pm 2, \ldots, i, j=1,2, \ldots, m$ suffices to define $E$-this follows easily by inspection. Then one finds, via the Reidemeister-Schreier procedure (see e.g., [15]), that the defining relations of $E^{*}$ are simply

$$
\begin{gathered}
{\left[\left(x^{-2}\right)^{t} a_{i, 0}\left(x^{2}\right)^{t}, a_{j, 0}\right]=1, \quad\left[\left(x^{-2}\right)^{t} a_{i, 1}\left(x^{2}\right)^{t}, a_{j, 0}\right]=1, \quad\left[\left(x^{-2}\right)^{t} a_{i, 1}\left(x^{2}\right)^{t}, a_{j, 1}\right]=1,} \\
{\left[\left(x^{-2}\right)^{t} a_{i, 0}\left(x^{2}\right)^{t}, z\right]=1, \quad\left[\left(x^{-2}\right)^{t} a_{i, 1}\left(x^{2}\right)^{t}, z\right]=1, \quad x^{-2} z x^{2}=a_{1,0}^{-3} a_{1,1}^{3} z .}
\end{gathered}
$$

Let us now consider $E^{*} / E_{2}^{*}$. The defining relations then give us $E^{*} / E_{2}^{*}$ as an abelian group on the generators

$$
x^{2}, a_{1,0}, \ldots, a_{m, 0}, a_{1,1}, \ldots, a_{m, 1}, z
$$

subject to the single defining relation

$$
a_{1,0}^{3}=a_{1,1}^{3} .
$$

It is therefore clear that $E^{*} / E_{2}^{*}$ cannot be generated by fewer than $2 m+2$ elements. Hence $E^{*}$ itself is a $(2 m+2)$-generator group.

4.7. We are left finally with the proof of Theorem 2 . For this it is certainly enough to prove that $G$ cannot be generated by $r$ elements. Now $\mathfrak{M}$ contains the variety of metabelian groups. Put $m=r-1$ and consider the group $E$ corresponding to this choice of $m$. Now $E$ is residually a finite 2-group. Therefore, in view of (10) and the Proposition 4, the mapping (cf. (3))

$$
\zeta: a \rightarrow x^{-1}, b \rightarrow x z, b^{\prime} \rightarrow a_{2,0}, \ldots, c \rightarrow a_{m, 0}
$$

can be continued to a homomorphism, again denoted by $\zeta$, of $G$ into $E$ taking $d$ to $a_{1,0}$. Obviously $\zeta$ is onto. Now Lemma 7 tells us that $G \zeta$ cannot be generated by fewer than $r+1$ elements. This completes the proof of Theorem 2 . 


\section{REFERENCES}

1. R. Baer and F. W. Levi, Freie Produkte und ihre Untergruppen, Math. Comp. 3 (1936), 391-398.

2. G. Baumslag, Some aspects of groups with unique roots, Acta Math. 104 (1960), 217-303.

3. - Some remarks on nilpotent groups with roots, Proc. Amer. Math. Soc. 12 (1961), 262-267.

4. - Residual nilpotence and relations in free groups, J. Algebra 2 (1965), 271-282.

5. - Groups with one defining relator, J. Austral. Math. Soc. 4 (1964), 385-392.

6. - On the residual nilpotence of some varietal products, Trans. Amer. Math. Soc. 109 (1963), 357-365.

7. G. Baumslag and K. W. Gruenberg, Some reflections on cohomological dimension and freeness, J. Algebra (1967), (to appear).

8. K. T. Chen, R. H. Fox and R. C. Lyndon, Free differential calculus. IV, Ann. of Math. 68 (1958), 81-95.

9. D. B. A. Epstein, A result on free products with amalgamations, J. London Math. Soc. 37 (1962), 130-132.

10. K. W. Gruenberg, Residual properties of infinite soluble groups, Proc. London Math. Soc.

(3) 7 (1957), 29-62.

11. - Lecture Notes, Queen Mary College, London, 1965-1966.

12. P. Hall, The splitting properties of relatively free groups, Proc. London Math. Soc. (3) 4 (1954), 343-356.

13. - Finiteness conditions for soluble groups, Proc. London Math. Soc. (3) 4 (1954), 419-436.

14. K. Iwasawa, Einige Sätze über freier Gruppen, Proc. Japan Acad. 19 (1963), 272-274.

15. A. Karass, W. Magnus and D. Solitar, Combinatorial group theory, Interscience, New York, 1965.

16. A. G. Kurosh, The theory of groups, Vol. 2, Chelsea, New York, 1955.

17. S. MacLane, Homology, Academic Press, New York, 1963.

18. W. Magnus, Beziehungen zwischen Gruppen und Idealen in einem speziellen Ring, Math. Ann. 111 (1935), 259-280.

19. - Uber freie Faktorgruppen und freie Untergruppen gegebener Gruppen, Monatsh. Math. Phys. 47 (1939), 307-313.

20. A. I. Mal'cev, Nilpotent torsion-free groups, Izv. Akad. Nauk SSSR Ser. Mat. 13 (1949), 201-212. (Russian)

21. - Generalized nilpotent algebras and their adjoint groups, Mat. Sb. 25 (1949), 347-366. (Russian)

22. H. Neumann, Generalized free products with amalgamated subgroups. II, Amer. J. Math. 71 (1949), 491-540.

23. J. Wiegold, Some remarks on generalized products of groups with amalgamations, Math. Z. 75 (1962), 57-78.

24. E. Witt, Treue Darstellung Liescher Ringe, J. Reine Angew. Math. 177 (1937), 152-160.

Graduate Center, City University of New York, New York, New York 